\title{
Stretchable Engineering Technologies for the development of advanced stretchable polymeric systems
}

\author{
F.AXISA, F. BOSSUYT, J. MISSINE, R. VERPLANCKE, T. VERVUST, J. VANFLETEREN, \\ IMEC-CMST, Ugent, Technology parc 914, Zwijnaarde, Belgium
}

\begin{abstract}
For advanced body related applications, there is a need of soft, conformable, elastic, mechanical compliant and washable systems. Smart clothes for health monitoring, sport or professional protection need washable and conformable electronic systems, which can be deformed up to $20 \%$. Implants, like monitoring sensors, or functional implants, need softness, stretchability to comply with the human body, chemical resistance, and biocompatibility. Many technologies are already available, like polyimide or PET/PEN based flexible electronic system, or conductive yarns for textile which can be knitted or embroidered to produce textrodes or textile based electronic systems. However softness and interconnections are still problems. Polymers based electronic system or stretchable electronic systems are a possible solution.

We have developed several technologies to produce stretchable electronics systems. They are based on the concept of flexible functional islands, onto which standard SMD components are soldered, interconnected with meanders shaped metallic stretchable interconnections. Metallic interconnections are made from copper or gold and are optimized using FEM analysis. Stretchable electronic systems are then molded in elastomeric (e.g. silicone rubber or polyurethane) matrix. They can sustain at least $100 \%$ of elongation and 3000 cycles at $20 \%$ of elongation. They can be integrated in textile and they are biocompatible and washable.
\end{abstract}

\section{Introduction}

Our daily life is more and more accompanied by electronic systems. Nowadays these systems show an increasing degree of complexity; however, this enhanced functionality may not lead to a decrease in comfort for the user: they evolve into wearable electronic system. They can be used anywhere, any time. It is clear that in this perspective, the electronics should preferably be conformable, and therefore flexible, or even better stretchable. Technologies are nowadays appearing for stretchable electronic: stretchable electronic board [6], stretchable microelectronic [5], conductive polymer [1]. Soft or stretchable technologies can also be used for medical application, like implantable device. It is possible to define a group of technologies, stretchable electronic technologies, which enable the design and the fabrication of complex stretchable system. This article presents different technologies to obtain reliable stretchable electronics system, as well as the basis of other stretchable technologies.

Stretchable Engineering Technologies (SET) can be defined as a coherent group of technologies to produce jelly type complex system, based on elastomer and flexible polymer. The potentials applications of SET are various: wearable electronic, moulded interconnect device packaging (MID), bio-implants and biocompatible packaging, robot, robotic skin, interface... For textiles, SET should provide technologies to soft and washable systems, compatible with textile process and technologies, in where electronics can be integrated in a stretchable, comfortable, 
conformable substrate. For bio-implants and biocompatible packaging, SET can provide technologies for soft or stretchable sensor and actuators. SET can be defined as a technology which integrates in a soft matrix electronic systems (including MEMS, battery, power management system, display, interfaces), RF communication systems, polymeric sensor and actuators, electro active polymer, micro fluidic systems, electro fluidic system (i.e. electro osmosis pump or electrophoresis based analysis system or actuators). The encapsulation polymer has physical properties (thermal conductivity, dielectric and electrical parameters, mechanical properties, optical properties, porosity, roughness, hydrophilicity) and chemical properties (color, permeability to gas or liquid) which can be functional. The encapsulant can be filled with different particles (nanoparticles, pigments, flakes...) to change the properties. The encapsulant can be engineered (molded, cast, plasma treated) and structure as a multilayer system..

\section{Stretchable electronic concept}

As electronic components are rigid or flexible, the main challenge to obtain stretchable electronic is to make stretchable interconnections between flexible or rigid groups of components, called functional islands. These stretchable interconnections links functional nonstretchable islands together in order to create stretchable electronic systems for biomedical applications, in which common SMD components, platinum plated electrodes, antennas and flexible batteries are incorporated. Despite the amelioration of conductive elastomer, conductivity is not comparable to metal, and they are also expensive. The best solution in term of conductivity and cost is still metal. Few research groups [3-6] have reported work on the development of stretchable metallic interconnections in elastic substrates. We propose an approach based on the one of Gray et al. [6] : The interconnection between two points is not a straight line but a twodimensional spring-shaped metal track.
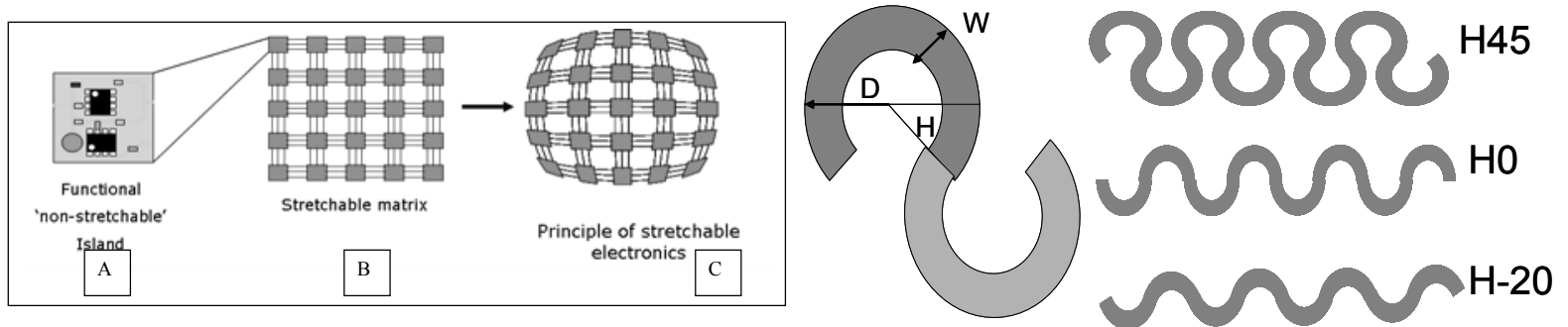

Figure 1 Stretchable electronic board is a matrix of rigid / flexible functional mini board on where components are soldered and interconnected with stretchable interconnections. Stretchable interconnections are formed with horseshoe shaped meander. Horseshoe shaped meanders are defined by their width $W$, their diameter $D$ and their angle $\mathrm{H}$. we note horseshoe with and angle of $0^{\circ} \mathrm{HO}$ and with an angle of $45^{\circ}, \mathrm{H} 45$.

The goal is to create stretchable interconnections which can support up to $100 \%$ elongation. Finite Element Analysis (FEA) has been used to characterize the shape of the conductors in order to allow high deformations without permanent damage [10]. Based on these results, a horseshoe shape was designed. Stresses are distributed in a wider region instead of being concentrated in a small zone. The horseshoe pattern is created by joining a series of circular arcs. Horseshoe shapes are defined by an angle, $\mathrm{H}$, a width $\mathrm{W}$ and a diameter D. Shape with an angle of 0 is called $\mathrm{H} 0$, shape with an angle of 45 is called H45, shape with an angle of -15 is called H-15. H45 has a high maximum elongation, but a higher electrical resistance. H0 has a lower maximum elongation, but a lower resistance. H-20 is stackable structure to reduce drastically the pitch. The length before elongation of an interconnection is $\lambda$ (length of the straight line between the two 
interconnection points). $\mathrm{L}$ is the length of the metal track (distance when following the meander shaped track). We can define several parameters to characterize a meander shape interconnection: The resistance per running length unit of the interconnection with $0 \%$ of elongation $r_{0},(\Omega / \mathrm{cm}) r_{0}$ is proportional to $\mathrm{L} / \lambda$. The maximum elongation of a conductive stretchable interconnection $\mathrm{E}_{\mathrm{max}}=100 *\left(\lambda_{\mathrm{F}}-\lambda_{0}\right) / \lambda_{0}$ where $\lambda_{\mathrm{F}}$ is the length at which the interconnection fails and $\lambda_{0}$ is the length without elongation The theoretical maximum elongation of an interconnection is expected to be proportional to the ratio $\mathrm{L} / \lambda$. Horseshoe shapes are periodic structures with a period of $2 \mathrm{x}$ $\cos (\mathrm{H}) \times(\mathrm{D}-\mathrm{W})$. Those structures are scalable. We define the aspect ratio as the $\mathrm{R}=\mathrm{P} / \mathrm{W}$. We also define the quality of an interconnection as $\mathrm{Q}=\mathrm{E}_{\max } / \mathrm{r}_{0}$. Q represents the efficiency of a stretchable pattern. For a constant ratio and a constant thickness of the meander, Q is also scalable.

\section{Technologies}

\subsection{Biocompatible process}

The general process to embed gold meander metallic interconnections into a stretchable material is shown in Figure 1. A photoresist is spin coated on a copper foil and patterned with the desired conductor shape. In step 3 of Figure 2 a nickel or platinum seed layer followed by a $4 \mu \mathrm{m}$ thick gold layer are electroplated. At last a Ni-Au finish is electroplated in order to be able to solder components on pad areas. As stretchable substrate material Silastic MDX4-4210 from Dow Corning has been used, which is a biomedical grade silicone elastomer. It has a low Young's modulus and a high elongation (470\%). In step 5 of Figure 2 the sample is overmoulded with the viscous $(\geq 60,000 \mathrm{cP})$ silicone. In step 7 of figure 2 the interconnection is embedded completely by applying another silicone layer. A stretchable electronic circuit can be made if thicker silicone areas at the soldered components can protect them against excessive strain. Silastic MDX4-4210 from Dow Corning is a biocompatible PDMS which can be used for medical application. However PDMS is hydrophobic polymer. For applications which need hydrophilic surfaces, an additional monolayer of vinyl monomer is chemically stitched to the surface.
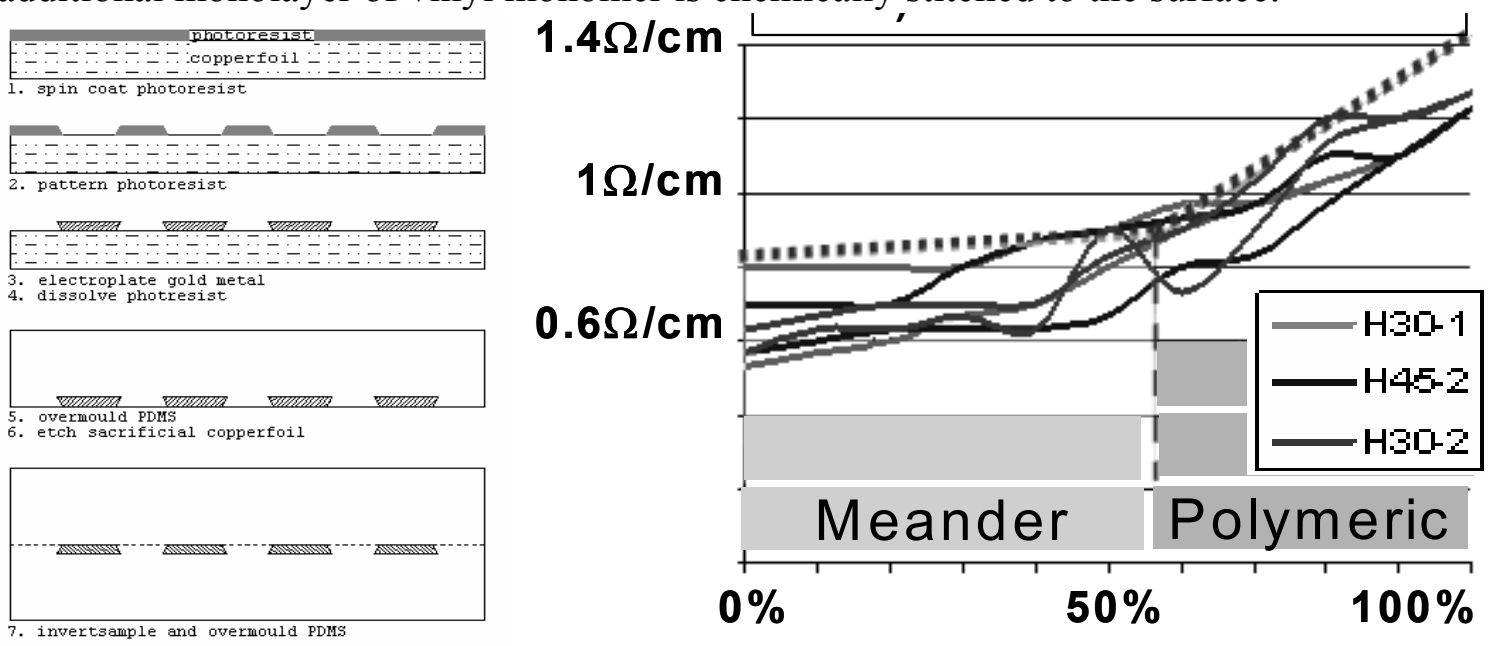

Figure 2. left side Process sequence for metallic stretchable interconnections embedded in PDMS. Right sideShunted meander's evolution of the resistivity under deformation of $100 \%$. 
$\mathrm{H} 0, \mathrm{H} 30$ and $\mathrm{H} 45$ have a very low increase of resistivity during elongation (below $2 \%$ of increase after mid elongation). Mean $\mathrm{E}_{\max }$ of $\mathrm{H0}$ is $57 \%$, of $\mathrm{H} 3075 \%$ and of $\mathrm{H} 4577 \%$. H0 has the higher quality (mean value $61 \mathrm{~cm} / \Omega$ ) and $\mathrm{H} 45$ has a significantly lower quality. In order to increase the reliability of stretchable electronic interconnections, meanders are shunted by deposition on the top of the metal meander of a layer of stretchable conductive polymer, which has lower conductivity but high elongation and reliability. If the metallic meander is broken, conductive polymer acts like a bridge or a shunt and ensure global connectivity. Conductive polymer can be deposited on a large band, or a narrow band, or multi narrow band, or in a meander shape. The polymer can be silver-filled polymer or organic conduction polymer (polypyrrole, Pedot, polyannilline, etc..), but should be stretchable. Figure 2 represents the evolution of linear resistivity of shunted meanders (meanders : H45, with a diameter of $450 \mu \mathrm{m}, \mathrm{Ni}-\mathrm{Au}-\mathrm{Ni}-\mathrm{Au}$;

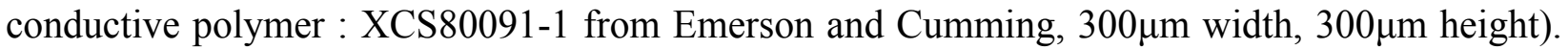
Without deformation, the resistivity is $0.7 \Omega / \mathrm{cm}$. Until a deformation of $60 \%$ the resistivity is less or equal to $0.8 \Omega / \mathrm{cm}$. Then the linear resistivity increase linearly to $1.2 \Omega / \mathrm{cm}$ under a deformation of $100 \%$. If the tension is released, the resistivity decreases linearly to $0.8 \Omega / \mathrm{cm}$ at $60 \%$ of deformation, and then goes back to $0.6 \Omega / \mathrm{cm}$. Until $60 \%$, the meander is intact, and then the whole structure behaves exactly like a simple meander. Beyond $60 \%$, cracks in the meander appear and the conductive polymer repairs it, which induces a larger increase of the resistivity.

\subsection{Fast prototyping process}

From any flexible substrate it is possible to obtain stretchable or partially stretchable system. The starting point is a flexible circuit on where components have been soldered or not.

At first the flexible (Fig 3.1) substrate is stitched to a support, using an adhesive (i.e. wax), on the entire surface or locally, the goal is to prevent the movement of the flexible board during processing and to ensure the flatness of the flexible (Fig 3.2). Then the meander shaped interconnections and the functional islands are cut using YAG laser. Its parameters are Nd-Yag laser $355 \mathrm{~nm},-375 \mathrm{~mW}, 10 \mathrm{kHz}$, ablation speed $5 \mathrm{~mm} / \mathrm{s}$, gaussian beam spot of +/- $20 \mathrm{um}$ diameters, angle: perpendicular.
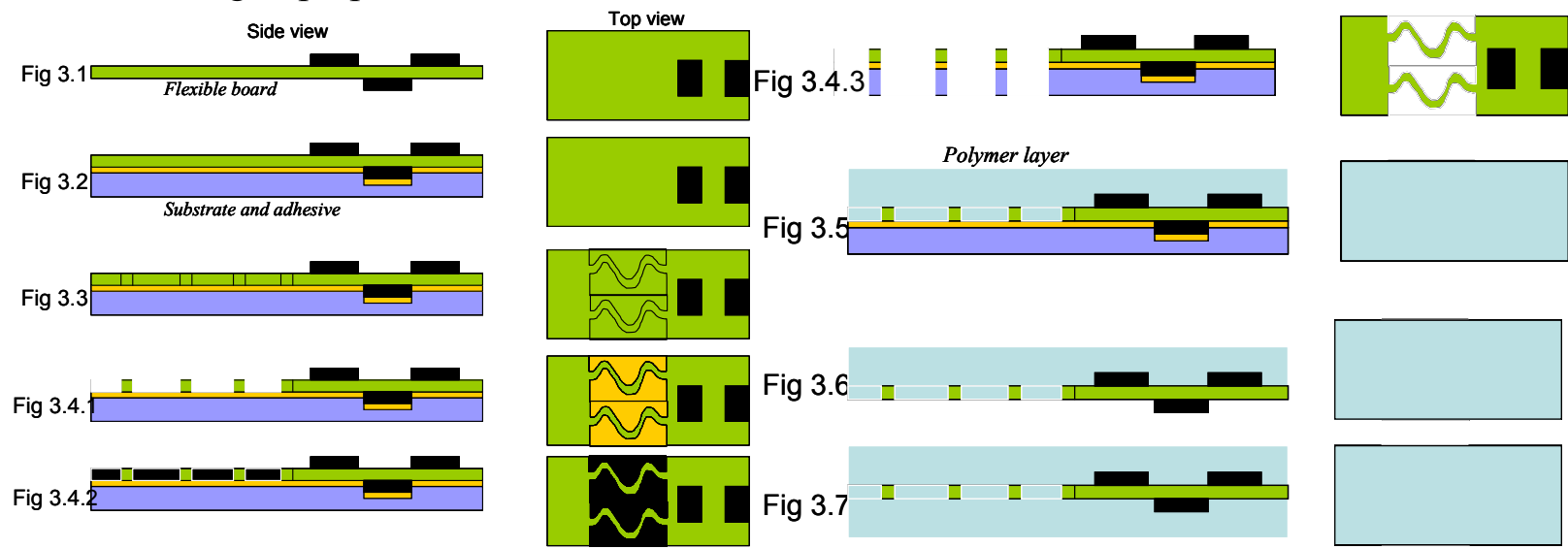

Figure 3 Process flow to obtain stretchable electronic board for a flexible board using YAG laser cutting technology and MID (Molded Interconnect device) for encapsulation.

The third step consists in removing the unused cut part of the flex (Fig 3.4.1). Then the flexible substrate is embedded in polymer using molding, casting or a laminating step (Fig 3.5). At this 
stage, the support can be already removed if the flex is rigid enough. After removing the support (dissolving the adhesive or melting the wax) (Fig 3.6) and, if needed, the circuit is embedded completely using molding or casting (Fig 3.7).The result is a system composed of flexible interposer connected together by conductive stretchable meanders, and all is embedded in polymer (Fig 3.7). This system is therefore stretchable and functional. This technology allows producing from any kind of flexible board a stretchable board. Complex multilayer stretchable electronic board can also be easily produced using this technology.

To provide a reliable way to embed components in a polymer, MID (Moulded Interconnect Device) strategy has been chosen. This technology consists in the encapsulation of the electronic system using a mould. The MID has 4 steps. Firstly, the substrate with meanders and soldered components is maintained in place. The upper layer of PDMS is then injected and cured. Secondly, the substrate is etched or removed. Then another injection plate is mounted on the mould to inject the final layer of PDMS. After curing this layer at $150^{\circ} \mathrm{C}$ during 30 minutes the system is unmoulded to obtain the stretchable system. The material chosen for the plates was the polyetherimide ULTEM $^{\circledR} 1000$, based on its broad chemical resistance, its high heat resistance and its high mechanical strength and rigidity.

\section{Demonstrators}

\section{1. actuators: stretchable heater}

As thermal actuator, a stretchable heater has been designed. The principle is to create three concentric resistive heating rings using stretchable meanders from stretchable electronic technology. Each ring provides a different amount of heat according to its electrical resistance. In order to diffuse the heat homogeneously, the system has a double layer of silicone.

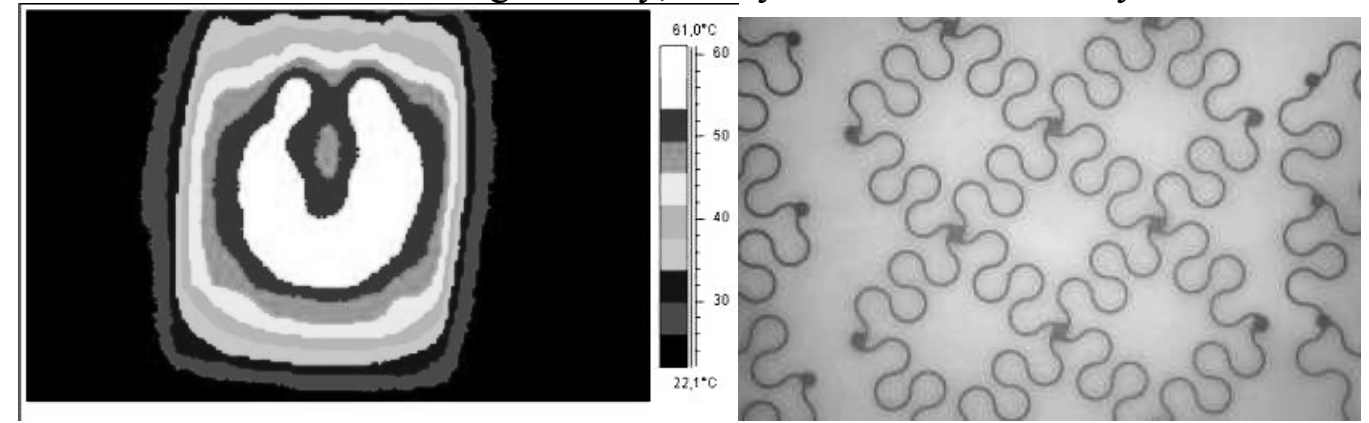

Figure 4. InfraRed view of the stretchable heater under a current of $2 A$ and detailed view of the stretchable heater: the second stretchable ring

The back face is made of thermally isolating silicone to direct the heat to the front face. The front is made of stretchable conductive silicone (3-6651 from Dow Corning), to diffuse the heat. The thickness of the conductive silicone layer and the fine tuning of the resistance of the three rings are parameters are optimized to equalize the heat diffusion.

\subsection{Led matrix in textile}

Fast prototyping technology was used for the processing of a simple matrix of 20 leds in a stretchable electronic system using this methodology and Figure 6 shows this matrix of 20 leds in a stretchable electronic system embedded in silicone rubber. This matrix is embedded in Sylgard 186 from DowCorning, and glued after low pressure plasma treatment to a textile impregnated with Sylgard 186. 


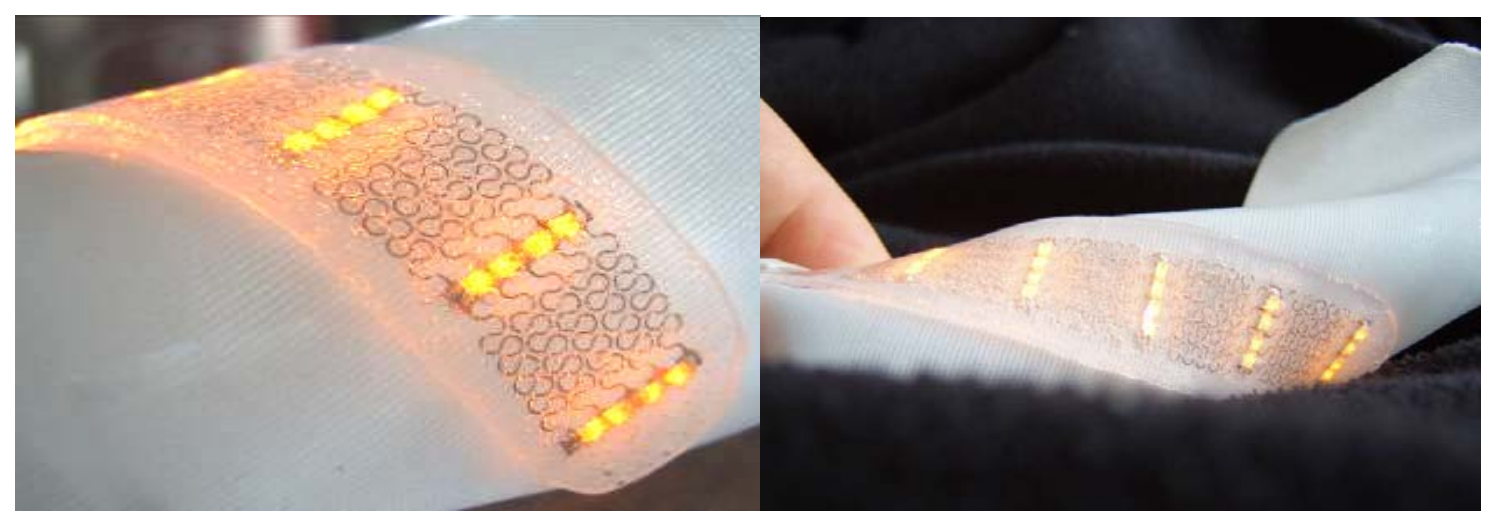

Figure 5 Textile on where a matrix of Led (20Leds) is encapsulated in Sylgard 186 (Dow Corning). The matrix is shaped using Yag Laser, encapsulated in silicone and plasma bonded on an impregnated textile with Sylgard186

\subsection{Stretchable watch and wireless inductive coil}

It is also possible to obtain a stretchable circuit formed with large flexible functional islands interconnected with laser shaped meander interconnections and embedded in silicone rubber. Figure 7 shows a stretchable watch with LCD display, microcontroller and battery, embedded in Sylgard 184 from Dow Corning. This watch is composed of flexible functional islands (based on polyimide substrate) interconnected by laser cut conductive meanders. Components are soldered a this stage (LCD display, battery, components). Then the watch is molded; A thick layer of silicone where components are, and a thin layer of silicone where interconnections are, in order to protect components from excessive mechanical stress.
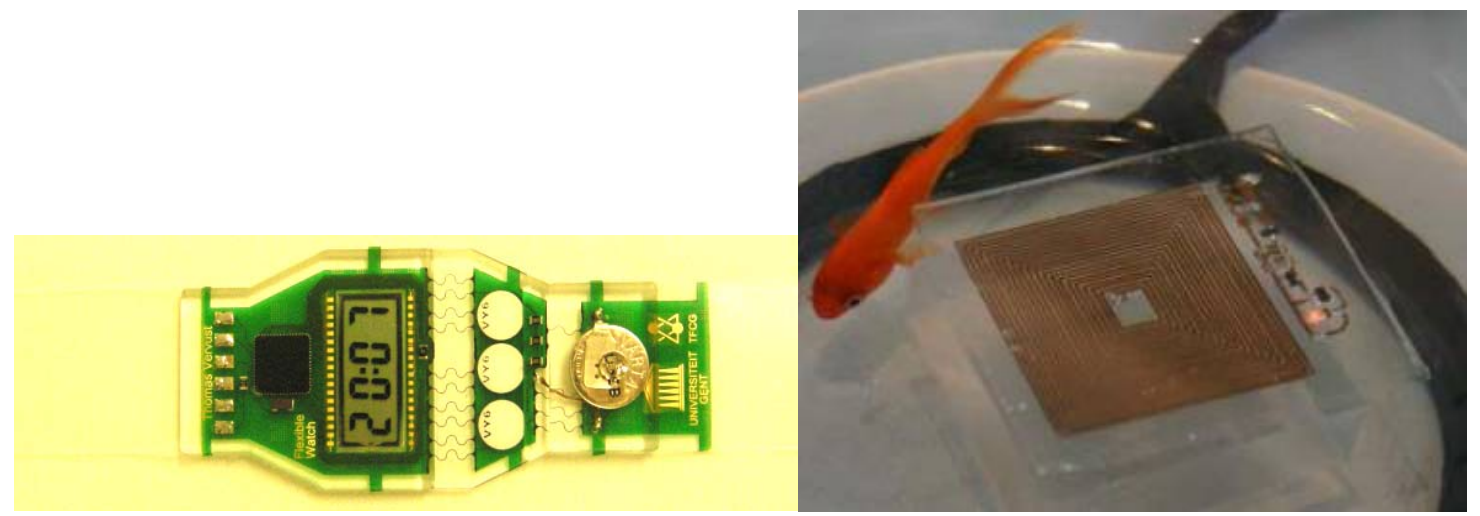

Figure 6 Left side Stretchable watch, composed of flexible board on where battery, micro-controller, LCD display, button have been soldered, interconnected with stretchable meander shape interconnection, and embedded in Sylgard 184. Right side: Inductive power coil in PDMS matrix in a fish pond

\section{Reliability testing}

In order to measure the reliability and the maximum elongation, a test vehicle had been designed. This test vehicle is composed of several lines; each line is composed of H45 meanders, and is therefore stretchable. The thickness of the meanders is $35 \mathrm{um}$ ( $25 \mathrm{um}$ of polyimide and $9 \mathrm{um}$ of copper). The width varies from $30 \mathrm{um}$ to $140 \mathrm{um}$ and the diameter from $500 \mathrm{um}$ to $900 \mathrm{um}$. The aspect ratio varies therefore from 5 to 17 . The first test consists in applying a periodic strain of $20 \%$ at a strain rate of $5 \%$ per second and to measure at the same time the conductivity. The test is finished when the interconnection is no more conductive. The test is performed on extension machine INSTRON 5543, which measure the elongation, the load and the electrical conductivity via 4 channels. Figure 8 represents the variation of the reliability of the meander according to the 
aspect ratio. Each point is the mean of 5 measurements. Under an aspect ratio of 8 , meanders appears bulky and the reliability is low. An optimum level is reached for an aspect ratio of 8 , for which the reliability is 3000 cycles. Above 8, the meanders become narrower, and are more sensitive to width variation due to the process: meanders become more fragile and the reliability decreases down to 1000 cycles for an aspect ratio above 14 .
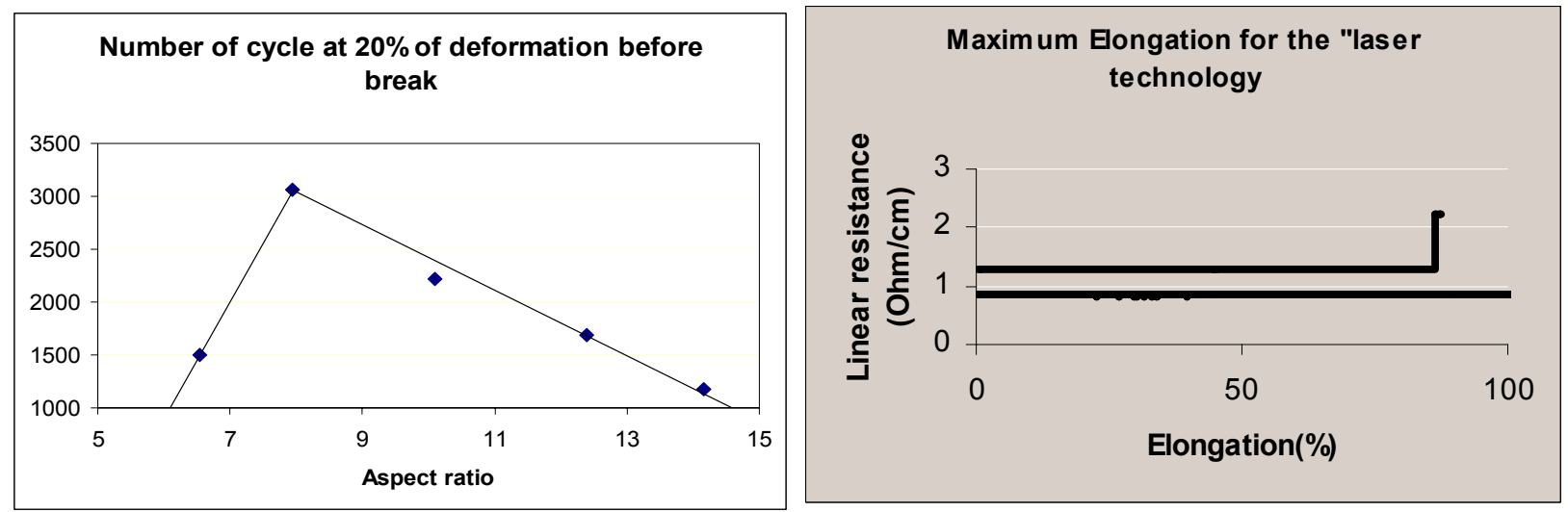

Figure 7 The number of cycle at 20\% of deformation depends of the aspect ratio. The optimal aspect ratio is 8 . For an aspect ratio of 8 , the number of cycle before break is 3000 cycles. The maximum elongation before break of the conduction obtained is above $100 \%$, the linear resistance is constant for any elongation and is about $10 \mathrm{hm} / \mathrm{cm}$.

A second test was performed to know the maximum elongation before break of the meanders and the variation of the resistance with elongation up to $100 \%$. Using the same measurement system, on elongation until break mode, we measured the linear resistance, defined par the resistance $\mathrm{R}$ divided by $\lambda(\mathrm{Ohm} / \mathrm{cm})$. The linear resistance varies from $0.8 \mathrm{Ohm} / \mathrm{cm}$ to $1.3 \mathrm{Ohm} / \mathrm{cm}$ depending of the aspect ratio. The maximum elongation varies from $80 \%$ to more than $100 \%$ without increase of the linear resistance.

\section{Conclusion : Concept of complex stretchable structure}
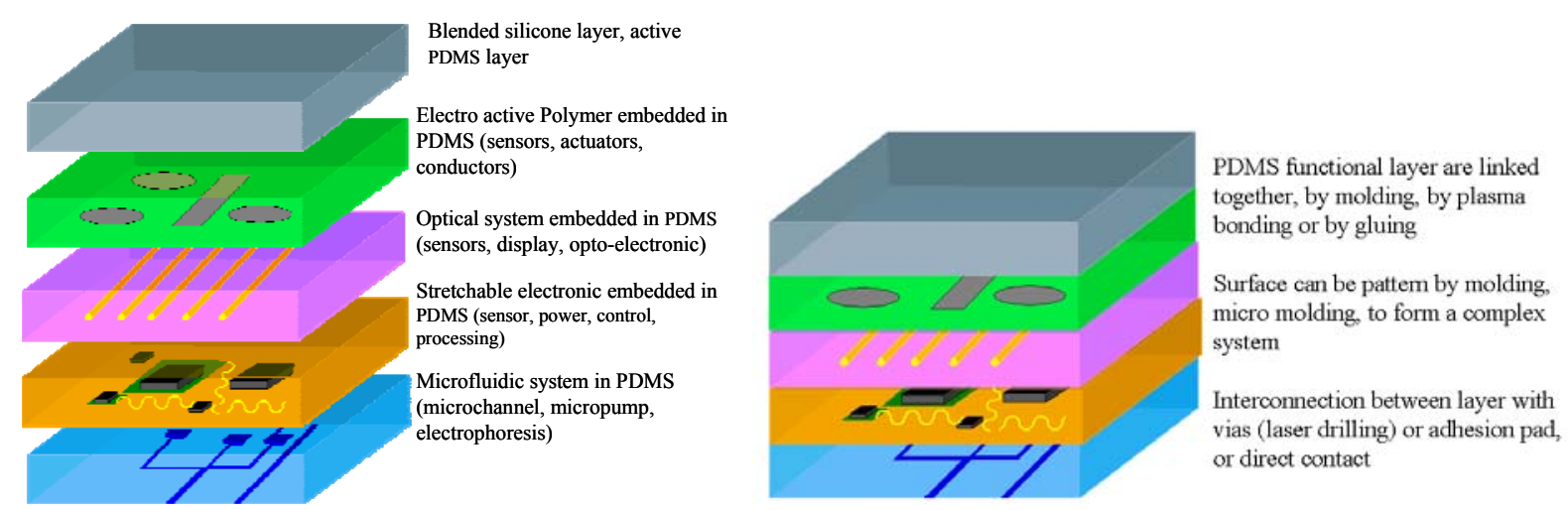

Figure 8 Final Concept of SET. Stretchable electronic system layer can be assemble with micro fluidic system layer and conductive polymer layer to form a complete stretchable systems for different applications.

A SET provides technologies to produces different types of stretchable system (stretchable electronic, micro-fluidic system, electro active polymer based system, optical system, blended elastomer membrane) and technologies to assemble those systems (molding, casting, plasma 
bonding). Those multilayer combined systems may have several applications: Biocompatible micro fluidic system controlled by embedded electronic system, washable electronic, robotic skin, soft robot...).

We have developed technologies to produce in a reliable way stretchable electronic system, with a maximum elongation up to $100 \%$ and a reliability of 3000 cycle at $20 \%$. It makes possible the creation of complex stretchable electronic circuit, combining flexible functional islands and stretchable interconnections in an elastomer matrix. It can be used for implants as well as for smart textiles. We are focusing on increasing the reliability of the stretchable board and developing our capabilities in processing microfluidic layer or electro-active polymer layer, in order to achieve and complete SET.

\section{Acknowledgement}

The work is supported by the Institute for the Promotion of Innovation by Science and Technology in Flanders (IWT) through the SBO-Bioflex project (contract number 04101). This work is also supported by European Commission Research programme STELLA (contract number 028026). This work is also supported by Belgium supported Research programme SWEET (belspo P2/08).

\section{References}

[1] F. Axisa, D. Brosteaux, E. De Leersnyder, F. Bossuyt, J. Vanfleteren, B. Hermans, and R. Puers, "Biomedical stretchable systems using MID based stretchable electronics technology," in Proceedings of the 29th Annual International Conference of the IEEE Engineering in Medicine and Biology Society, (Lyon, France), pp. 56875690, August 2007.

[2] Fabrice Axisa, D. Brosteaux, E. De Leersnyder, F. Bossuyt, M. Gonzalez, M. Vanden Bulcke, J. Vanfleteren, Elastic and Conformable Electronic Circuits and Assemblies Using MID in Polymer, Polytronics 2007, $6^{\text {th }}$ international IEEE conference polymers and adhesives in Microelectronics and Photonics, p280-286, 2007

[3] D. Brosteaux, F. Axisa, M. Gonzalez, and J. Vanfleteren, "Design and fabrication of elastic interconnections for stretchable electronic circuits," IEEE Electron Device Letters, vol. 28, no. 7, pp. 552-554, 2007.

[4] T. Loher, D. Manessis, R. Heinrich, B. Schmied, J. Vanfleteren, J. De Baets, A. Ostmann, and H. Reichl, "Stretchable electronic systems," in Proceedings of the 8th Electronics Packaging Technolgy Conference (EPTC), (Singapore), pp. 271-276, December 2006.

[5] Lacour S.P., Jones J., Suo Z., Wagner S., "Design and Performance of Thin Metal Film Interconnects for SkinLike Electronic Circuits,” IEEE Electron Device Letters,Vol. 25, No. 4 (2004), pp. 179-181

[6] D. S. Gray, J. Tien, and C. S. Chen, "High-conductivity elastomeric electronics," Adv. Mater., vol. 16, no. 5, pp. 393-397, Mar. 2004.

[7] STELLA IST 028086 FP6 European Project : Development of stretchable electronics for large area applications for use in healthcare, wellness and functional clothes, integrated electronics in stretchable parts and products. http://www.stella-project.de/

[8] SWEET Stretchable Washable Electronics for Embedding in Textiles, Belspo P2/08, http://www.belspo.be/belspo/fedra/proj.asp?l=en\&COD $=\mathrm{P} 2 / 08$

http://tfcg.elis.ugent.be/projects/sweet/index.html

[9] BIOFLEX, Biocompatible Flexible Electronic Circuits, IWT-SBO, IWT-040101, http://trappist.elis.ugent.be/elisgroups/tfcg/projects/bioflex/Welcome.html

[10] Mario Gonzalez, Fabrice Axisa, Mathieu Vanden Bulcke, Dominique Brosteaux, Bart Vandevelde and Jan Vanfleteren," Design of Metal Interconnects for Stretchable Electronic Circuits using Finite Element Analysis", 8th. Int. Conf. on Thermal, Mechanical and Multiphysics Simulation and Experiments in Micro-Electronics and Micro-Systems, EuroSimE 2007 